\title{
THE EQUIVARIANT EXTENSION THEOREM
}

\author{
R. LASHOF
}

\begin{abstract}
A simplified proof of Jaworowski's equivariant extension theorem is given which enables one to generalize the domain to a class of $G$-spaces which include all (not necessarily compact) $G$-manifolds.
\end{abstract}

Let $G$ be a compact Lie group. In [2] Jaworowski proved the Extension Theorem: Let $X$ be a locally compact separable metric finite-dimensonal $G$-space with a finite number of orbit types. Let $A$ be a closed invariant subspace and let $f: A \rightarrow Y$ be a $G$-map to a locally compact metric separable $G$-space $Y$. If $Y^{H}$ is an ANR (resp. AR) for every orbit type $(H)$ in $X-A$, then $f$ has a neighborhood $G$-extension (resp. a $G$-extension over $X$ ).

The purpose of this note is to give a simplified proof of the extension theorem which will enable us to extend Jaworowski's result to a class of $G$-spaces $X$ which include all (not necessarily compact) $G$-manifolds.

The following lemma is proved in Steenrod [4] for the case $Y$ is an AR; the case where $Y$ is an ANR requires only minor revisions.

LEMMA 1. Let $p: E \rightarrow X$ be a locally trivial fibre bundle with fibre $Y$ an $A N R$ $(A R)$. Suppose $X$ is a normal Lindelöf space and $A \subset X$ a closed subspace. If $s$ : $A \rightarrow p^{-1}(A)$ is a section over $A$, then $s$ can be extended to a section over $a$ neighborhood of $A$ (over $X$ ).

Lemma 2 generalizes the well-known result on principal bundle maps, and may be proved in the same way, cf. Kosniowski [3].

LEMMA 2. Let $X$ be a completely regular free $G$-space and $Y$ an arbitrary $G$-space. Then equivariant maps of $X$ into $Y$ are in bijective correspondence with cross-sections of the associated bundle $\varphi: X \times_{G} Y \rightarrow X / G$ with fibre $Y$ to the principal $G$-bundle $q: X \rightarrow X / G$.

LEMMA 3. Let $A$ be a closed subspace of the topological space $X$. Let $Y$ be a metric space and $Z$ a compact space. Let $f: A \rightarrow Y$ and $g: X-A \rightarrow Z$ be continuous functions. If $\varphi: X \times Z \rightarrow Y$ is a continuous extension of $f \circ \pi_{1}, \pi_{1}: A \times Z \rightarrow A$, the projection onto the first coordinate, then the function $F: X \rightarrow Y, F|A=f, F| X-A$ $=\varphi \circ(i, g), i: X-A \rightarrow X$ the inclusion, is a continuous extension of $f$.

Recieved by the editors April 21, 1980 and, in revised form, June 6, 1980.

1980 Mathematics Subject Classification. Primary 55M15, 57S10, 57S15.

(C) American Mathematical Society 0002-9939/81/0000-0431/\$01.75 
Proof. Since $X-A$ is open, we need only prove continuity at points of $A$. If $a \in A$ and $\varepsilon>0$, then, since $Z$ is compact, there is a neighborhood $U$ of $a$ in $X$ such that $d\left(\varphi_{x}, \varphi_{a}\right)<\varepsilon, x \in U, \varphi_{x}: Z \rightarrow Y$, and $\varphi_{x}(z)=\varphi(x, z)$. In particular, if $x \in U \cap(X-A), d(\varphi(x, g(x)), \varphi(a, g(x)))<\varepsilon$. But $\varphi(a, g(x))=f(a)$. We can also choose $U$ sufficiently small so that $x$ in $U \cap A$ implies $d(f(x), f(a))<\varepsilon$. Hence for $x \in U, d(F(x), F(a))<\varepsilon$. Thus $F$ is continuous.

REMARK. If $X, Y$ and $Z$ are $G$-spaces, $A$ is invariant and $f, g$ and $\varphi$ are equivariant, then $F$ is equivariant.

The following lemma is proved, for example, in Bredon [1].

LEMMA 4. For every $n$, there is a compact $n$-universal G-bundle. (Here $n$-universal means that it classifies $G$-bundles over paracompact spaces of covering dimension $<n$.)

MaIn Lemma. Let $X$ be a separable metric G-space and $A$ a closed invariant subspace such that $X-A$ is finite dimensional with a single orbit type $(H)$. Suppose $f: A \rightarrow Y$ is an equivariant map into a metrizable $G$-space. If $Y^{H}$ is an $A N R(A R)$, then $f$ extends to an equivariant map on a neighborhood of $A$ (on $X$ ).

Proof. Let $X_{H}$ be the points of orbit type $(H)$ and let $X_{H}=X_{(H)} \cap X^{H}$. Then [1], $X_{(H)}=G \times_{N(H)} X_{H}=G / H \times_{K} X_{H}, K=N(H) / H$. Hence a $G$-map of $X_{(H)}$ into $Y$ is completely determined by a $K$-map of $X_{H}$ into $Y^{H}$. Since $X-A \subset X_{(H)}$, to extend $f$ it is sufficient to extend the $K$-map $f^{H}: A^{H} \rightarrow Y^{H}$ to a neighborhood of $A^{H}$ in $X^{H}$ (to $X^{H}$ ). In fact, since $X^{H}$ is closed in $X$ and $G \times X \rightarrow X$ is closed, $G X^{H}$ is the quotient space of $G \times X^{H}$. Thus, if $F^{H}$ extends $f^{H}$ to a neighborhood $U$ of $A^{H}$ (to $X^{H}$ ), the map $F: G U \rightarrow Y\left(G X^{H} \rightarrow Y\right), F(g x)=g F^{H}(x)$ is continuous and agrees with $f$ on $G U \cap A\left(G X^{H} \cap A\right)$ and hence $f$ extends to a neighborhood of $A$ (to $X=G X^{H} \cup A$ ).

Let $Z$ be a compact $n$-universal $K$-bundle, $n \geqslant \operatorname{dim}(X-A) / G$. Then, on the one hand, $X^{H} \times Z$ is a free $K$-space and, on the other, we have a $K$-bundle map of $X^{H}-A^{H}$ to $Z$. Now by Lemmas 1 and $2, f^{H} \circ \pi_{1}: A \times Z \rightarrow Y^{H}$ extends to a $K$-map $\varphi$ of $U \times Z, U$ a neighborhood of $A^{H}$ (of $X^{H} \times Z$ ) into $Y$. By Lemma 3, this gives a $K$-extension of $f^{H}$ to $U$ (to $X^{H}$ ); and $f$ extends to a neighborhood of $A$ (to $X$ ).

Theorem A. Let $X$ be a separable metric $G$-space and $A$ a closed invariant subspace such that $X-A$ is finite dimensional with a finite number of orbit types. Suppose $f: A \rightarrow Y$ is an equivariant map into a metrizable $G$-space. If $Y^{H}$ is an ANR $(A R)$ for each orbit type $(H)$ in $X-A$, then $f$ extends to an equivariant map on $a$ neighborhood of $A$ (on $X$ ).

Proof. Partially order the orbit types $\left(H_{1}\right),\left(H_{2}\right), \ldots,\left(H_{r}\right)$ in $X-A$ so that if $\left(H_{i}\right) \geqslant\left(H_{j}\right)$ then $i \geqslant j$. Let $X_{i}=\cup_{j<i} X_{\left(H_{j}\right)}$. Then $X=A \cup X_{r}$. We inductively assume we have an equivariant extension of $f$ to $W_{i-1}, W_{i-1}$ an invariant neighborhood of $A$ in $A \cup X_{i-1}$ (to $A \cup X_{i-1}$ ). Then $W_{i-1} \cup X_{\left(H_{i}\right)}-W_{i-1}$ (resp. $A \cup X_{i}-$ $\left.A \cup X_{i-1}\right)$ has a single orbit type and since closure $\left(X_{i-1}\right)$ includes only points of 
orbit type $<\left(H_{j}\right), j \leqslant i-1, W_{i-1}$ (resp. $\left.A \cup X_{i-1}\right)$ is closed in $W_{i-1} \cup X_{\left(H_{i}\right)}$ (resp. $\left.A \cup X_{i}\right)$. By the Main Theorem, $f$ extends to a neighborhood $W_{i}$ of $W_{i-1}$ in $W_{i-1} \cup X_{\left(H_{i}\right)}\left(\right.$ resp. $\left.A \cup X_{i}\right)$. Since

$$
W_{i-1} \cup X_{\left(H_{i}\right)}=A \cup X_{i}-\left(X_{i-1}-W_{i-1}\right)
$$

is a neighborhood of $A$ in $A \cup X_{i}$, so is $W_{i}$. Thus we have proved the inductive step and the result follows.

Definition. A $G$-space $X$ has locally finite orbit structure if each orbit in $X$ has a neighborhood with only finitely many orbit types.

Let $A$ be an invariant subspace of $X$. A $G$-space $X$ has localy finite orbit structure $\bmod A$ if each orbit $G(x)$ in $X$ has a neighborhood $U_{x}$ with only finitely many orbit types in $U_{x}-A$.

Definition. A separable metric space $X$ has locally finite dimension if each $x \in X$ has a finite-dimensional neighborhood.

Let $A$ be a subspace of $X . X$ has locally finite dimension mod $A$ if each $x \in X$ has a neighborhood $U_{x}$ such that $U_{x}-A$ has finite dimension.

REMARK. A $G$-manifold has locally finite orbit structure and locally finite dimension (cf. Chapters VI and VII of Borel's Seminar on Transformation Groups, Ann. of Math. Studies, no. 46, Princeton Univ. Press, Princeton, N. J., 1960).

Theorem B. Let $X$ be a separable metric $G$-space and $A$ a closed invariant subspace such that $X$ has locally finite dimension and locally finite orbit structure mod A. Suppose $f: A \rightarrow Y$ is an equivariant map into a metrizable $G$-space. If each $Y^{H}$, $(H)$ an orbit type of $X-A$, is an $A N R(A R)$, then $f$ extends to an equivariant map on a neighborhood of $A$ (on $X$ ).

Proof. Since $X$ is normal, every neighborhood of an orbit contains a closed invariant neighborhood. Since $X$ is Lindelöf, we can choose a countable number of these neighborhoods, $V_{1}, V_{2}, \ldots$ such that $\cup \operatorname{Int} V_{i}=X$, and each $V_{i}-A$ has only finitely many orbit types and is finite dimensional. Let $X^{i}=U_{j<i} V_{j}$. Then $X^{i}$ is closed, and $\cup \operatorname{Int} X^{i}=X$. By induction, it is sufficient to prove the result for $A^{i} \subset X^{i}, A^{i}=\left(A \cap X^{i}\right) \cup W^{i-1}$ (resp. $\left.\left(A \cap X^{i}\right) \cup X^{i-1}\right)$, when we assume $f$ has been extended to $A \cup W^{i-1}$ (resp. $A \cup X^{i-1}$ ), where $W^{i-1}$ is a neighborhood of $A \cap X^{i-1}$ in $X^{i-1}$. But $X^{i}-A^{i}$ has only finitely many orbit types and is finite dimensional. Hence, the inductive step follows from Theorem A, proving Theorem B.

\section{REFERENCES}

1. G. Bredon Indtroduction to compact transformation groups. Academic Press, New York, 1972.

2. Jan Jaworowski, Extension of G-maps and Euclidean G-retracts, Math. Z. 146 (1976), 143-148.

3. C. Kosniowski, Equivariant cohomology and stable cohomotopy, Math. Ann. 210 (1974), 83-104.

4. N. Steenrod, The topology of fibre bundles, Princeton Math. Series, vol. 14, Princeton Univ. Press, Princeton, N. J., 1951.

Department of Mathematics, University of Chicago, Chicago, Illinois 60637 